\title{
Study on Aggregation of Inductive Motor Loads Based on Differential Evolution Algorithm
}

\author{
Baozhen Zhang ${ }^{1}$ and Chenxi Fan ${ }^{2}$ \\ ${ }^{1}$ School of Mechanical and Electrical Engineering, Hainan University, Haikou, China \\ ${ }^{2}$ School of Electric Power, South China University of Technology, Guangzhou, China
}

\begin{abstract}
In order to simplify enormous inductive-motor loads in online estimation dynamic equivalence for large-scale power systems, it is presented identification strategy based on the algorithm of differential evolution algorithm (DE) to acquired the parameter of equivalent motor loads. It presented to dynamic aggregate motors into an equivalent motor based on same or similar dynamic characteristic, the parameter of equivalent motor is identified to use modified Differential Evolution algorithm (DE) which has unique advantage in nonlinear parameter optimization, in order to solve premature convergence problem of DE, double evolution swarm which adopt different variation are formed. Simulated results indicate that it is high accuracy for identified the parameter of equivalent motors, equivalent system can preserve dynamic characteristics of original system well. The method can be used to simplify mass inductive motor loads in online estimation dynamic equivalence for large-scale power systems.
\end{abstract}

Keywords-power system; estimation dynamic equivalence; inductive-motor load; differential evolution; parameter identification

\section{INTRODUCTION}

The method of estimation dynamic equivalence is used to reducing large-scale power systems for online dynamic safety analysis aim, this dynamic equivalence method is that external system is presented using equivalent system based on real-time online measured quantity [1]. It is necessary that mass inductive-motor loads should also taken into account in estimation dynamic equivalence except static loads, parameters of induction-motor loads have significance influence on simulation precision of power systems, it is proved that the recall fault simulation for two blackouts in west American [2, 3]. If mass inductive-motor loads existed in external system can be partition based on characteristic, we can aggregate those motor loads for an equivalent motor load, while those motor loads have same or similar characteristic, the equivalent accuracy is also improved.

The literatures studying on reducing inductive-motor loads are not more. We can be grouping inductive-motor loads based on their same or similar characteristic [4]. In literature [5], the cluster method is presented to identify motor load group, and also the parameter of equivalent motor load is acquired using the weighting method. In literature [6], motor loads are grouped with fuzzy- $c$ clustering method, then, the parameter of the equivalent motor which aggregate into the individual motor in the same group is obtained by average-weighting method. Because of enormous induction-motor loads in large-scale power systems, it is difficult to get the parameter of inductionmotor load model in detailed if we take into account calculation quantity. In order to solve this problem, identification strategy is employed to aggregate inductive motor loads which have same or similar characteristic [7], the algorithms are used to identification the parameter of induction-motor load [8,9], such as Genetic Algorithm (GA), chaos optimization, etc.. On account of the parameter identification for non-liner system based on optimization algorithm, therefore, it is necessary to find suited algorithm which has excellent property to be satisfied with rapid and accuracy requirement in online estimation dynamic equivalence.

In order to solve the equivalent simplification problem for mass inductive-motor loads, firstly, the model of inductivemotor load is given. Secondly, identification strategy is presented to acquire the parameter of equivalent inductivemotor loads, Thirdly, it is presented Differential Evolution algorithm (DE) and improvement measure to identification the parameter of equivalent inductive-motor loads. Finally, simulation test is given to prove the validity of modified DE algorithm.

\section{MODEL OF INDUCTION-MTOTR}

In order to describe the dynamic performance of inductionmotor better, the 3rd order model based on electromechanical transient is adopted. The equivalent circuit of induction-motor is shown in Fig. 1, where $r_{s}, X_{s}$ and $r_{r}^{\prime}, X_{r}^{\prime}$ are the equivalent resistance and leakage reactance of stator and rotor, respectively; $r_{m}$ and $X_{m}$ are the resistance and reactance of excitation branch, respectively.

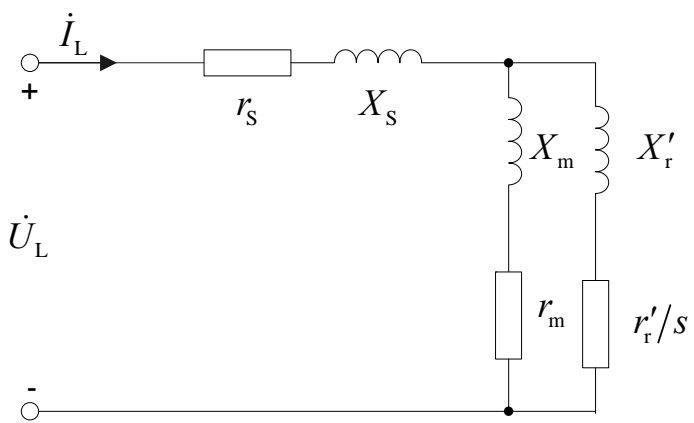

FIGURE I. EQUIVALENT CIRCUIT OF INDUCTIVE-MOTOR MODEL

Eq. (1) is the equations for the 3rd order model based on electromechanical transient for inductive-motor [10]. 


$$
\left\{\begin{array}{l}
\dot{U}=\dot{E}^{\prime}+\left(r_{\mathrm{s}}+j X^{\prime}\right) \dot{I} \\
p \dot{E}^{\prime}=-j s \dot{E}^{\prime}-\frac{1}{T^{\prime}}\left[\dot{E}^{\prime}-j\left(X-X^{\prime}\right) \dot{I}\right] \\
T_{\mathrm{J}} \frac{d s}{s t}=T_{\mathrm{m}}-T_{\mathrm{e}}
\end{array}\right.
$$

where $U$ and $E^{\prime}$ are the voltage and transient potential; $X$ is the open-circuit reactance of rotor, and $X=X_{s}+X_{m} ; X^{\prime}$ is the transient reactance, and $X^{\prime}=X_{s}+X_{m} / / X_{r} ; T^{\prime}$ is the open loop time-constant of stator, and $T^{\prime}=\left(X_{r}+X_{m}\right) / r_{r} ; T_{m}$ is the mechanical torque, and $T_{m}=T_{0}\left(A \omega^{2}+B \omega^{+} C\right) / r_{r}, A \omega^{2}+B \omega+C=1 ; T_{0}$ and $T_{e}$ are the mechanical torque of steady state and electromagnetism torque, respectively; $T_{J}$ is the inertia time-constant; $p$ is the differential operator.

When identification strategy is adopted, eight parameter are preparative identification optimized for inductive-motor load, they are $\left[r_{s}, X_{s}, X_{m}, r_{r}, X_{r}, T_{\mathrm{J}}, A, B\right]$ (we can think $r_{m}$ equal zero based on reference [1]).

\section{IDENTIFICATION STRATEGY OF MOTOR' PARAMETER}

The objection function $J_{m}$ is constructed when we identify the parameter of equivalent inductive-motor [11], $J_{m}$ is calculated by Eq. (2). Block diagram of identification the equivalent inductive-motor parameter is shown in Fig. 2.

$$
J_{m}=\sqrt{\sum_{i=1}^{M}\left(P_{1}-P_{2}\right)^{2}+\left(Q_{1}-Q_{2}\right)^{2}}
$$

where $P_{1}$ and $Q_{1}$ are the active power and reactive power of equivalent system; ; $P_{2}$ and $Q_{2}$ are the active power and reactive power of actual system; $M$ is the number of data in simulation process.

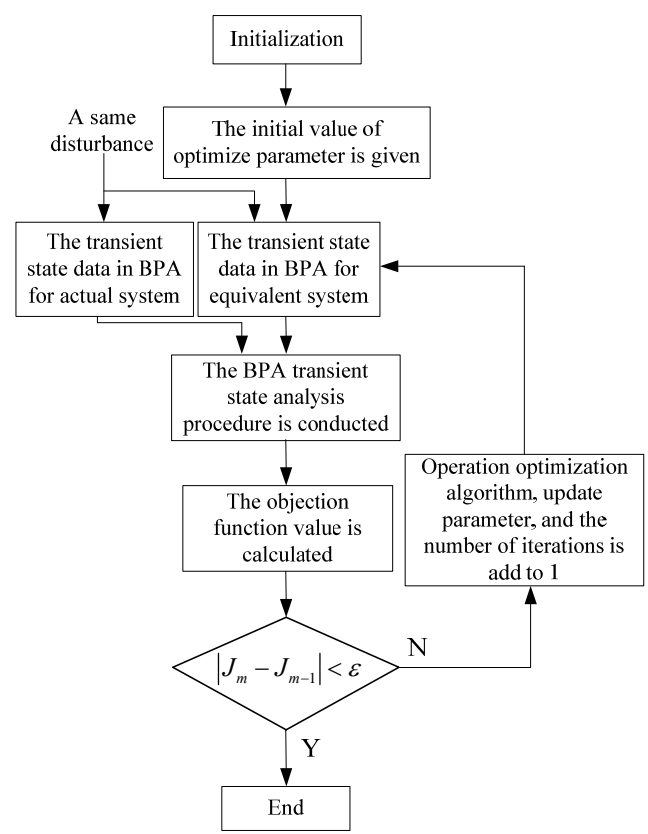

FIGURE II. BLOCK DIAGRAM OF IDENTIFICATION THE EQUIVALENT MOTOR PARAMETER

\section{DifFERENTIAL EVOLUTION ALGORITHM}

\section{A. The Basic Differential Evolution Algorithm}

Differential Evolution algorithm (DE) is a newer evolution algorithm that was proposed in 1995, the optimization process is operated by the way of "black box" in DE, which do not demand graded information, continuity and differentiability for the function, DE algorithm is suitable for the problem that optimizes non-liner parameter and multi-variable coupling optimization $[12,13]$.

It includes three steps during the optimized process of evolution in DE, namely, mutation, crossover and selection operation. There are various kinds $\mathrm{DE}$ due to difference mutation ways, DE/rand/1/bin and DE/best/2/bin are the most popular way. The mutation step of $\mathrm{DE} / \mathrm{rand} / 1 / \mathrm{bin}$ and DE/best/2/bin are operated by Eq. (3) and Eq. (4), the crossover and selection step are operated by Eq. (5) and Eq. (6).

$$
\begin{gathered}
\boldsymbol{y}_{i}{ }^{t+1}=\mathbf{z}_{i}{ }^{t}+(k R)\left(\mathbf{z}_{s 1}{ }^{t}-\mathbf{z}_{s 2}{ }^{t}\right) \\
\boldsymbol{y}_{i}{ }^{(t+1)}=\mathbf{z}_{\text {gbest }}{ }^{t}+(k R)\left[\left(\mathbf{z}_{\mathrm{s} 1}{ }^{t}-\mathbf{z}_{\mathrm{s} 2}{ }^{t}\right)+\left(\mathbf{z}_{\mathrm{s} 3}{ }^{t}-\mathbf{z}_{\mathrm{s} 4}{ }^{t}\right)\right] \\
R_{i j}{ }^{t+1}= \begin{cases}y_{i j}{ }^{t+1}, & \text { rand }(j) \leq(C R) \\
z_{i j}{ }^{t}, & \text { otherwise }\end{cases} \\
\mathbf{z}_{i}{ }^{t+1}= \begin{cases}\boldsymbol{R}_{i}{ }^{t+1}, & f\left(\boldsymbol{R}_{i}^{t+1}\right)<f\left(\mathbf{z}_{i}{ }^{t}\right) \\
\mathbf{z}_{i}{ }^{t}, & f\left(\boldsymbol{R}_{i}{ }^{t+1}\right) \geq f\left(\mathbf{z}_{i}{ }^{t}\right)\end{cases}
\end{gathered}
$$

where $\mathbf{z}_{s 1}, \quad \mathbf{z}_{s 2}, \quad \mathbf{z}_{s 3}$ and $\mathbf{z}_{s 4}$ are the different individual in DE swarm, and $s 1, s 2, s 3, s 4$ are not equal to $i ; \mathbf{z}_{i}^{t}$ is target vector; $\mathbf{z}_{\boldsymbol{g} b e s t}$ is optimizing vector at present; $\left(\mathbf{z}_{s 1}{ }^{t}-\mathbf{z}_{s 2}{ }^{t}\right)$ and $\left(\mathbf{z}_{s 3}{ }^{t}-\mathbf{z}_{s 4}{ }^{t}\right)$ are differential vector; $(k R)$ is zoom factor; $\boldsymbol{y}_{i}^{t+1}$ is mutation vector; $R_{i j}{ }^{t+1}$ is the $j$-th dimension of test vector $\boldsymbol{R}_{i}^{t+1} ; \operatorname{rand}(j)$ is rand number, its range is $[0,1]$; $(C R)$ is crossover probability factor; $t$ is the number of iterations for evolutional generation.

\section{B. Modified DE}

The disadvantage of basic DE is that search stagnation and premature convergence phenomenon, $\mathrm{DE} / \mathrm{rand} / 1 /$ bin has better global searching ability, DE/best/2/bin has better local searching ability, if we can combine $\mathrm{DE} / \mathrm{rand} / 1 / \mathrm{bin}$ with DE/best/2/bin, the modified DE has better robustness, performance of convergence speed and stability [14]. The block diagram of modified DE algorithm is shown in Fig. 3.

\begin{tabular}{|l|l|}
\hline \multicolumn{1}{|c|}{ The first swarm } \\
1. Random initialization; \\
2. Each individual fitness value is \\
calculated; \\
3. The mutation way of DE/rand/1/bin second swarm \\
is adopted; \\
4. The crossover operation is \\
conducted; \\
$\begin{array}{l}\text { 5. The selection operation is } \\
\text { conducted; } \\
\text { 6. Repeat the steps of } 2-5 \text { until to } \\
\text { convergence. }\end{array}$
\end{tabular}

FIGURE III. BLOCK DIAGRAM OF MODIFIED DE 
The immigrant is adopted after every swarm evolving five generation independent in Fig. 3, the objective function $J_{m}$ is defined as the fitness function of modified DE. The procedure steps of modified DE algorithm are shown as follows.

- Initialization;

- The immigrant is operation after every swarm evolve five generation independent, the immigrant principle is that the worst individual of first swarm is replaced by the optimal individual of second swarm;

- In order to evaluate good and bad for per individual, The objective function is calculated by Eq. (2), the fitness value of per individual is equal to the objective function value;

- The mutation operation of two swarms are conducted by Eq. (3) and Eq. (4);

- The crossover operation of two swarms are conducted by Eq. (5);

- The selection operation of two swarms are conducted by Eq. (6);

- Inspection end condition, namely, the difference value of objective function of twice iteration whether less than a small given-positive number $\varepsilon$, calculation is end if the end condition is satisfied, and then to step (8); otherwise, the loop procedure of step (2) to step (6) is operated;

- End, output results.

\section{SimUlation ANALYSis}

The diagram of IEEE 10-generators and 39-buses system is shown in Fig. 4, the system is divided into two parts by the border of 39, 3 and 27 bus, the upper of dotted line is reserved, others loads are reduced. The data of inductive motor loads is completely consistent with the reference [15].

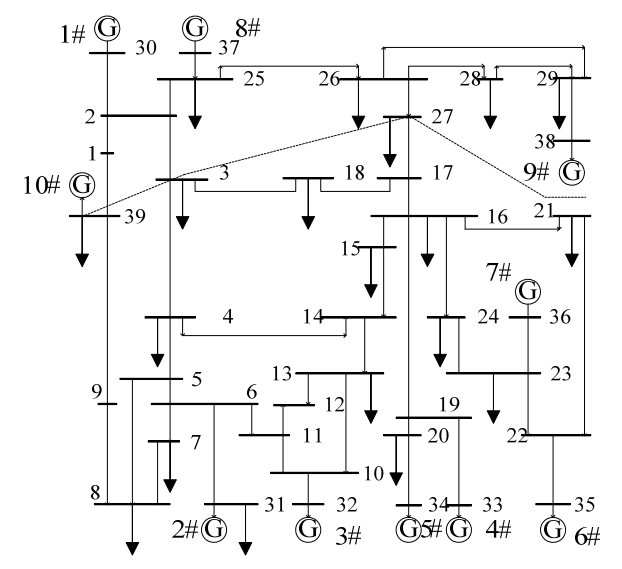

FIGURE IV. DIAGRAM OF IEEE 10-GENERATORS

There are thirteen loads in reduced system, the group result of inductive motor loads refer to reference [5], the thirteen loads is divided into two groups, the first group is $3,4,7,8,12$, 15 and 18, the second group is $16,20,21,23,24$ and 27. For a example, we study the reduced problem on 16, 20, 21, 23, 24 and 27 loads, six inductive-motor loads which include 16, 20, 21, 23, 24 and 27 are aggregated into a equivalent motor load $\mathrm{E}_{\mathrm{L}}$ using identification strategy based on modified $\mathrm{DE}$, the equivalent motor load $E_{\mathrm{L}}$ is connect with bus 16 [5], it is shown in Fig. 5.

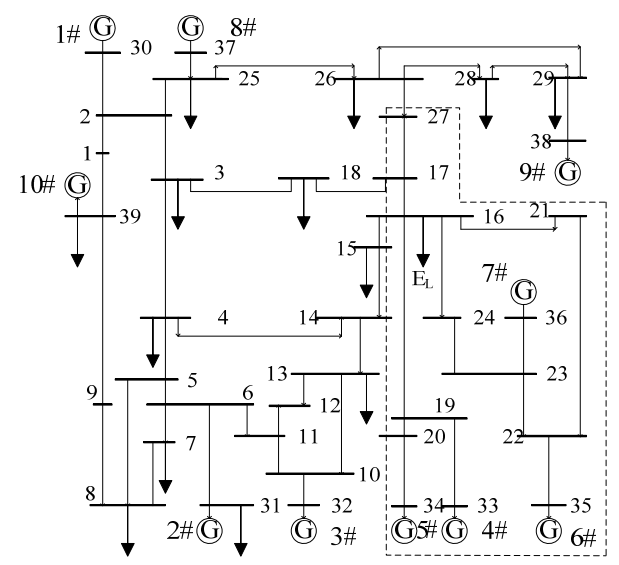

FIGURE V. DIAGRAM OF EQUIVALENT SYSTEM

The modified DE is used to identify the parameter of equivalent load $E_{\mathrm{L}}$, which the eight parameter are $r_{s}, X_{s}, X_{m}, r_{r}, X_{r}, T_{\mathrm{J}}, A, B$. The factor $(k R)$ and $(C R)$ are 0.6 and 0.9 in modified DE, respectively, the swarm scale is $5 \sim 10$ times waiting identification parameter. The three-phase fault was occurred on 9-39 line and cleared after 0.12 second and simulated for 12 second based on BPA software in the power systems of Fig. 4 and Fig. 5. Parameter of equivalent load $E_{L}$ is shown in Tab. 1.

TABLE I. PARAMETER OF EQUIVALENT LOAD E $\mathrm{E}_{\mathrm{L}}$

\begin{tabular}{ccccccccc}
\hline parameter & $r_{s}$ & $X_{s}$ & $X_{m}$ & $r_{r}$ & $X_{r}$ & $A$ & $B$ & $T_{J}$ \\
\hline $\mathrm{EL}_{\mathrm{L}}$ & 0.12 & 0.14 & 2.08 & 0.13 & 0.13 & 0.50 & 0.50 & 2.00 \\
\hline
\end{tabular}

For the sake of demonstration the accuracy of the dynamic responses of the equivalent load, a three-phase short-circuit fault was occurred on 9-39 line and cleared after 0.12 second and simulated for 12 second based on BPA software. The fault is applied on the original system as well as on the equivalent system.

We make analysis to several crucial elements, such as $10 \#$ generator because of close to fault local, 1 bus because of close to fault local, 14-15 line because of close to equivalent area. Transient responses curves that the swing curves of 10\# generator, the bus voltage of 1 bus, the active power flow of 14-15 line and reactive power flow of 14-15 line obtained from the simulations are presented in Fig. 6-9.

From these curves, the dynamic response of equivalent system is close to the original system, the tendency of curves change is consistent, basically. The simulation results presents that the dynamic characteristic of the original system has preserved in the equivalent system, and also it presents the accuracy of identified the parameter for equivalent inductivemotor load. 


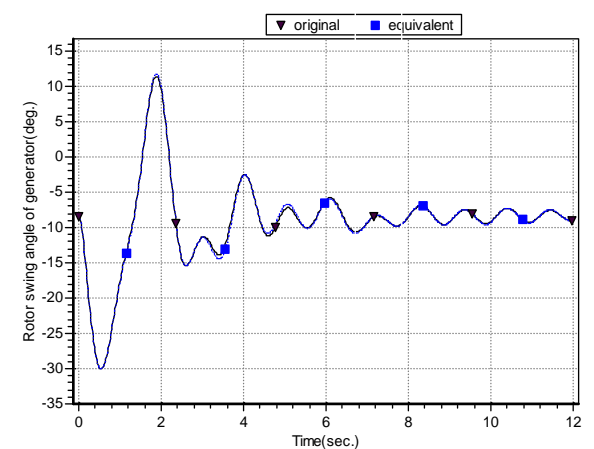

FIGURE VI. SWING CURVES OF 10\# GENERATOR

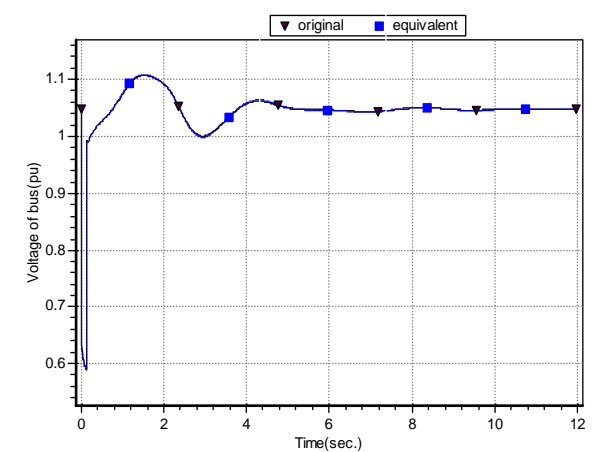

FIGURE VII. SWING CURVES OF 10\# GENERATOR

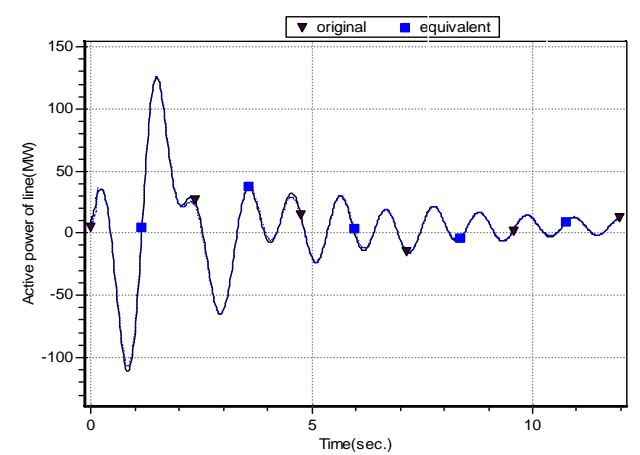

FIGURE VIII. AVTIVE POWER FLOW OF 14-15 LINE

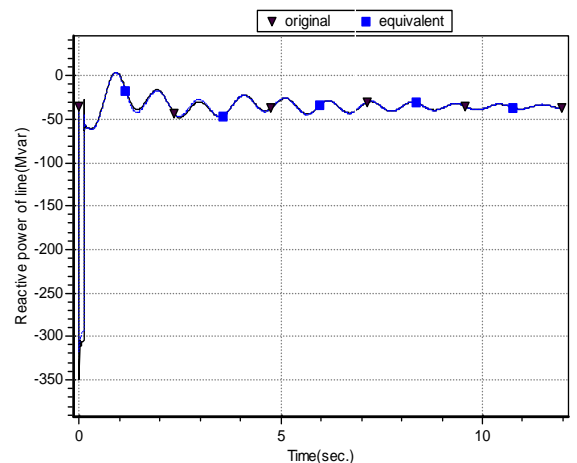

FIGURE IX. REAVTIVE POWER FLOW OF 14-15 LINE

\section{CONCLUSIONS}

The problem that identified the parameter of inductivemotor load is solved well by means of using modified DE algorithm, the shortcoming of basic DE algorithm which falls into local optimum are preferably improved, modified DE algorithm has better convergence performance, the parameter of equivalent inductive-motor load has higher precision, the main characteristics of the original system are preserved in the equivalent system. Modified DE algorithm has lower computation costs so that it is realized easily, identification strategy is suitable for reducing inductive-motor loads in online estimation dynamic equivalence for large-scale power systems because it is simplicity, high efficiency.

\section{ACKNOWLEDGMENT}

This work is supported by Hainan Province Nature Science Fund (No.20155205).

\section{REFERENCES}

[1] Yinxin Ni, Shousun Chen, Baolin Zhang, Dynamic power systems theroy and its analysis, Tsinghua university press, 2002, pp. 330-333.

[2] Jin Ma, Dong Han, He Ren-Mu, et al., "Reducing identified parameters of measurement-based composite load model”, IEEE Transactions on Power Systems, vol. 23, No. 1, pp. 76-83, 2008.

[3] Zhang Bao-zhen, Study on Dynamic Equivalence Method and Related Technology for Large Scale Power Systems, Ph.D. thesis, pp. 3-6, South China University of Technology, 2013.

[4] Zhang Jian, Sun Yuan-zhang, "Generalized load modeling considering wind generators connected to distribution network", Power System Technology, vol. 35, No. 8, pp. 41-46, 2011.

[5] Zhou Hai-qiang, Ju Ping, Yang hui, et al., "The Gynamic Equivalent Method for Large-scale Power Systems Considering the Motor Load”, China Science: Technology Science, vol. 40, pp. 704-710, 2010.

[6] Zhang Jian, Sun Yuan-zhang, Xu Jian, et al., “A dynamic aggregation method for induction motors based on their coherent characteristics", Automation of Electric Power Systems, vol. 34, No. 5, pp. 48-52, 2010.

[7] Shen Shan-de, Power systems identification, Tsinghua university press, 1993, pp. 1-20.

[8] Hua Bai, Pei Zhang, Venkataramana Ajjarapu, "A novel parameter identification approach via hybrid learning for aggregate load modeling”, IEEE Transactions on Power Systems, vol. 24, No. 3, pp. 1145-1154, 2009.

[9] Wang Shuang-xin, Jiang Yan, Han Fang, “A chaotic optimization strategy used in parameter identification of composite load model”, Proceedings of the CSEE, vol. 26, No. 12, pp. 111-116, 2006.

[10] Ju Ping, Ma Da-qiang, The load model for power systems, China power press, 2008, pp. 32-50.

[11] Zhang Bao-zhen, Zhang Yao, Lin Ling-xue, "Estimation Equivalence Method Based on Modified Differential Evolution Algorithm”, Journal of South China University of Technology: Natural Science Edition, vol. 42, No. 4, pp. 7-12, 2014.

[12] Storn R, Price K. Differential evolution - a simple and efficient adaptive scheme for global optimization over continuous spaces, Berkley: Technical Report International Computer Science Institute, pp.1-3,1995.

[13] Rainer S, Price K, "Differential evolution - a simple and efficient heuristic for global optimization over continuous spaces”, Journal of Global Optimization, vol. 11, No. 4, pp. 341-359, 1997.

[14] Wu Liang-hong, The Research and Applications of Differential Evolution Algorithm, M.D. thesis, pp. 56-60, Hunan University, 2007.

[15] Tang Yong, He Ren-mu, Ju Ping, et al., Dynamic character and security analysis for power receiver-side systems, Tsinghua university press, pp. 183-194, 2010. 